\title{
The Two-Dimensional Quantum Heisenberg Antiferromagnet with Ising-Like Anisotropy
}

\author{
Alessandro Cuccoli, Valerio Tognetti, Tommaso Roscilde, Paola Verrucchi, \\ Dipartimento di Fisica dell'Università di Firenze \\ and Istituto Nazionale di Fisica della Materia (INFM), \\ Largo E. Fermi 2, I-50125 Firenze, Italy \\ and Ruggero Vaia \\ Istituto di Elettronica Quantistica del Consiglio Nazionale delle Ricerche, \\ via Panciatichi 56/30, I-50127 Firenze, Italy, \\ and Istituto Nazionale di Fisica della Materia (INFM)
}

Received on 5 August, 2000

\begin{abstract}
We study the two dimensional quantum Heisenberg antiferromagnet on the square lattice with easyaxis exchange anisotropy by the semiclassical method called pure-quantum self-consistent harmonic approximation. In particular, we focus on the problem of the existence of a finite-temperature transition in such a model, and study the corresponding critical temperature as the spin value and the anisotropy vary. We find that an Ising-like transition characterizes the model even when the anisotropy is of the order of $10^{-2} J$ ( $J$ being the intra-layer exchange integral). The good agreement found between our theoretical results and the experimental data for the compounds $\mathrm{Rb}_{2} \mathrm{MnF}_{4}$, $\mathrm{K}_{2} \mathrm{MnF}_{4}$, and $\mathrm{K}_{2} \mathrm{NiF}_{4}$ shows that the insertion of the easy-axis exchange anisotropy, with quantum effects properly taken into account, provides a quantitative description and explanation of the real system's critical behaviour.
\end{abstract}

The easy-axis quantum Heisenberg antiferromagnet on the square lattice (EA-QHAF) is defined by the following Hamiltonian:

$$
\hat{\mathcal{H}}=\frac{J}{2} \sum_{\mathbf{i}, \mathbf{d}}\left[\mu\left(\hat{S}_{\mathbf{i}}^{x} \hat{S}_{\mathbf{i}+\mathbf{d}}^{x}+\hat{S}_{\mathbf{i}}^{y} \hat{S}_{\mathbf{i}+\mathbf{d}}^{y}\right)+\hat{S}_{\mathbf{i}}^{z} \hat{S}_{\mathbf{i}+\mathbf{d}}^{z}\right]
$$

where $\mathbf{i}=\left(i_{1}, i_{2}\right)$ runs over the sites of a square lattice, d connects each site to its four nearest neighbours, $J>0$ is the antiferromagnetic exchange integral and the anisotropy parameter $\mu$ takes values $\mu \in[0,1)$ for easy-axis models. The spin operators $\hat{S}_{\mathbf{i}}^{\alpha}$ $(\alpha=x, y, z)$ are such that $|\hat{\mathbf{S}}|^{2}=S(S+1)$ and obey $\left[\hat{S}_{\mathbf{i}}^{\alpha}, \hat{S}_{\mathbf{j}}^{\beta}\right]=i \varepsilon_{\alpha \beta \gamma} \delta_{\mathbf{i j}} \hat{S}_{\mathbf{i}}^{\gamma}$.

The anisotropy parameter $\mu$ measures the strength of the easy-axis character of the model: For $\mu=0$ the Ising-limit (equivalent to the Ising model if $S=1 / 2$ ) is recovered; in the opposite limit, Eq. (1) with $\mu=1$ describes the fully isotropic QHAF. At a classical level the EA-HAF is known to share its critical behaviour with the Ising model [1], with a finite-temperature secondorder phase transition corresponding to the onset of Nèel antiferromagnetic order. The critical temperature $T_{\mathrm{c}}^{\mathrm{cl}}$ is a decreasing function of $\mu$, and vanishes logarith- mically in the isotropic limit [2].

When quantum effects are considered, one expects the critical behaviour of the model to remain the same, as the universality class of the system cannot be changed by short-ranged quantum fluctuations [3]; however, these may be responsible for an evident lowering of the critical temperature, as quantum disorder opposes the onset of Neèl order. In principle, this effect could be strong enough to push the critical temperature down to $T_{\mathrm{c}}=0$, thus destroying the transition itself; the parameter region where such a scenario could possibly occur is that of low critical temperatures $(\mu \simeq 1)$ and strong quantum effects $(S=1 / 2)[4]$.

To address the problem of the existence of a finite temperature transition in the EA-QHAF, one needs to analyse the $\mu$-dependence of the critical temperature for different spins; results relative to precise spin and anisotropy values, typical output of quantum numerical simulations, are hence insufficient, albeit useful. The main goal of this paper is in fact to determine the critical temperature of the EA-QHAF as a function of both $\mu$ and $S$, and compare our theoretical results with the available experimental data. 
There exist several real compounds whose magnetic behaviour [5] is well described by the Hamiltonian Eq. (1); most of them, despite being characterized by a very weak easy-axis anisotropy $(\mu \simeq 1)$, show a finite-temperature phase-transition. Whether the transition is due to the anisotropy-driven onset of twodimensional Neèl order or to some other effect (such as the inter-layer exchange interaction), is not easily detectable experimentally, but strong indications in favour of the first possibility derive from the analysis of several thermodynamic quantities. This work confirms such a picture, as the critical temperatures relative to the compounds $\mathrm{Rb}_{2} \mathrm{MnF}_{4}$ [6] $(S=5 / 2, \mu=0.994)$, $\mathrm{K}_{2} \mathrm{MnF}_{4}$ [7] $(S=5 / 2, \mu=0.995)$ and $\mathrm{K}_{2} \mathrm{NiF}_{4}$ [8] ( $S=1, \mu=0.996$ ) agree nicely with the theoretical results for the two-dimensional EA-QHAF.

In order to study the thermodynamic and critical behaviour of the model described by Eq. (1), we use the pure-quantum self-consistent harmonic approximation (PQSCHA) [9]: this approximation allows one to take into account exactly the full classical, and the linear quantum contributions to the thermodynamics of the system, thus approximating (one-loop level) the non-linear pure-quantum contribution only. As the latter plays a secondary role in determining the critical properties of models with a not-too-strong quantum character, the PQSCHA is an ideal tool to study the thermodynamic and critical behaviour of many quantum systems, as shown by its many successful appli- cations [10], in particular to low-dimensional magnetic systems $[11,12]$.

The method, based on the path-integral formulation of quantum statistical mechanics, allows one to write statistical averages of quantum operators in the form of classical-like expressions: These may then be evaluated by classical (possibly numerical) techniques, like the transfer-matrix method in one dimension, or classical Monte Carlo simulation in two dimensions. In greater detail, the PQSCHA expression for the statistical average of a of quantum operator $\hat{O}$, for a magnetic system $N$ spins, reads

$$
\langle\hat{O}\rangle=\frac{1}{\mathcal{Z}_{\mathrm{eff}}} \int d^{N} \mathbf{s} O_{\mathrm{eff}} e^{-\beta \mathcal{H}_{\mathrm{eff}}}
$$

where $\beta=T^{-1}, \mathcal{Z}_{\text {eff }}=\int d^{N} \mathbf{s} e^{-\beta \mathcal{H}_{\text {eff }}}, \mathbf{s}=\left(s^{x}, s^{y}, s^{z}\right)$ is a classical unit vector, and $\int d^{N} \mathbf{s}=\Pi_{\mathbf{i}=1}^{N} \int d \mathbf{s}_{\mathbf{i}}$.

Quantum effects come into play via the spin and temperature dependence of $\mathcal{H}_{\text {eff }}$ and $O_{\text {eff }}$ : this dependence relates the original quantum system to an infinite class of classical easy-axis models, each defined by different parameters. This means that the PQSCHA reduces the study of quantum statistical averages to the evaluation of classical-like effective averages defined by Eq. (2), for to effective models which are different for different temperatures and spin values.

The specific PQSCHA expression for the effective Hamiltonian of the EA-QHAF is found to be

$$
\begin{aligned}
\mathcal{H}_{\mathrm{eff}} & =-\frac{1}{2} J \widetilde{S}^{2} \sum_{\mathbf{i}, \mathbf{d}}\left[\theta_{\perp}^{4} \mu\left(s_{\mathbf{i}}^{x} s_{\mathbf{i}+\mathbf{d}}^{x}+s_{\mathbf{i}}^{y} s_{\mathbf{i}+\mathbf{d}}^{y}\right)+\theta_{\|}^{2} \theta_{\perp}^{2} s_{\mathbf{i}}^{z} s_{\mathbf{i}+\mathbf{d}}^{z}\right] \\
& +\mathcal{G}(t, \widetilde{S})
\end{aligned}
$$

where, $\widetilde{S}=S+1 / 2$, and $t=T / J \widetilde{S}^{2}$ is the reduced temperature used hereafter.

The renormalization parameters are

$$
\theta_{\|}^{2}=1-\frac{\mathcal{D}_{\|}}{2} \quad, \quad \theta_{\perp}^{2}=1-\frac{\mathcal{D}_{\perp}}{2},
$$

where the coefficients

$$
\begin{aligned}
\mathcal{D}_{\|} & =\frac{1}{N \widetilde{S}} \sum_{\mathbf{k}} \frac{a_{\mathbf{k}}}{b_{\mathbf{k}}}\left(1-\mu \gamma_{\mathbf{k}}\right) \mathcal{L}_{\mathbf{k}}, \\
\mathcal{D}_{\perp} & =\frac{1}{N \widetilde{S}} \sum_{\mathbf{k}} \frac{a_{\mathbf{k}}}{b_{\mathbf{k}}}\left(1-\frac{\gamma_{\mathbf{k}}}{\mu}\right) \mathcal{L}_{\mathbf{k}}
\end{aligned}
$$

are self-consistently determined by solving Eqs. (4) and (5), with

$$
a_{\mathbf{k}}^{2}=4\left(\theta_{\|}^{2}+\mu \theta_{\perp}^{2} \gamma_{\mathbf{k}}\right), b_{\mathbf{k}}^{2}=4\left(\theta_{\|}^{2}-\mu \theta_{\perp}^{2} \gamma_{\mathbf{k}}\right),
$$

and

$\gamma_{\mathbf{k}}=\frac{1}{4} \sum_{\mathbf{d}} e^{i \mathbf{k} \cdot \mathbf{d}}, \quad \mathcal{L}_{\mathbf{k}}=\operatorname{coth} f_{\mathbf{k}}-\frac{1}{f_{\mathbf{k}}}, \quad f_{\mathbf{k}}=\frac{a_{\mathbf{k}} b_{\mathbf{k}}}{2 \widetilde{S} t}$,

$\mathbf{k}$ being the wave vector in the first Brillouin zone.

The temperature and spin dependent uniform term

$$
\mathcal{G}(t, \widetilde{S})=2 J \widetilde{S}^{2}\left(1-\theta_{\|}^{2} \theta_{\perp}^{2}\right)+J \widetilde{S}^{2} t \sum_{\mathbf{k}} \ln \left(\frac{\sinh f_{\mathbf{k}}}{\theta_{\perp}^{2} f_{\mathbf{k}}}\right),
$$

does not enter the expressions for the statistical averages, but contributes to the free energy and to the related thermodynamic quantities.

If one defines the effective exchange integral and effective anisotropy,

$$
J_{\mathrm{eff}}=J \theta_{\|}^{2} \theta_{\perp}^{2} \quad, \quad \mu_{\mathrm{eff}}=\mu \frac{\theta_{\perp}^{2}}{\theta_{\|}^{2}},
$$


Eq. (3) can be written in the form

$$
\begin{aligned}
\mathcal{H}_{\mathrm{eff}} & =-\frac{1}{2} J_{\mathrm{eff}} \widetilde{S}^{2} \sum_{\mathbf{i}, \mathbf{d}}\left[\mu_{\mathrm{eff}}\left(s_{\mathbf{i}}^{x} s_{\mathbf{i}+\mathbf{d}}^{x}+s_{\mathbf{i}}^{y} s_{\mathbf{i}+\mathbf{d}}^{y}\right)+s_{\mathbf{i}}^{z} s_{\mathbf{i}+\mathbf{d}}^{z}\right] \\
& +\mathcal{G}(t, \widetilde{S})
\end{aligned}
$$

showing that the thermodynamic behaviour of the EAQHAF is that of a classical EA-HAF with exchange integral $J_{\text {eff }}(t, S)$ and anisotropy parameter $\mu_{\text {eff }}(t, S)$. As $J_{\text {eff }}<J$ and $\mu_{\text {eff }}>\mu$ we see that quantum fluctuations induce a softening of both the energy scale and the anisotropy, thus inducing a higher degree of disorder in the system.

In Fig. 1 we show $j_{\text {eff }}=J_{\text {eff }} / J$ as a function of $t$, for different spin and anisotropy values: Quantum effects are markedly stronger for lower spins, while the $\mu$ dependence of the energy scale renormalization is seen to be extremely weak.

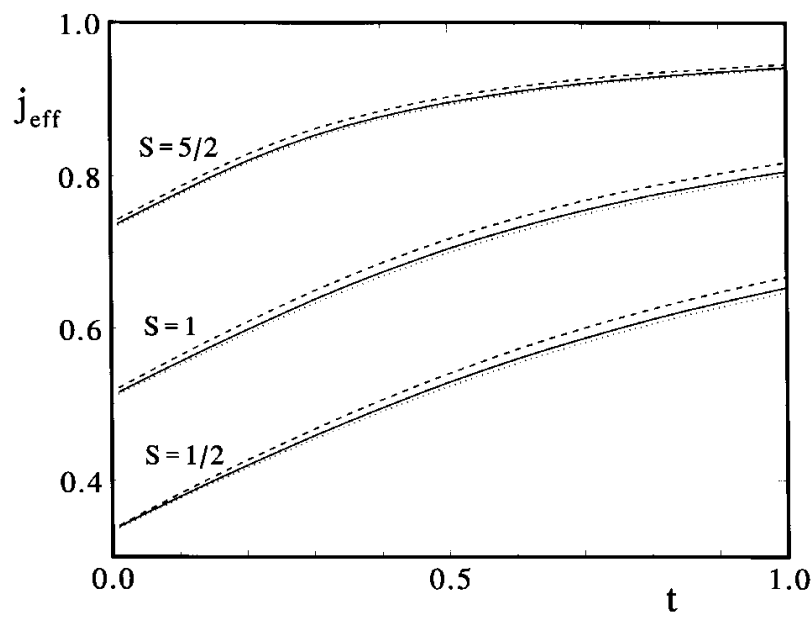

Figure 1. Renormalized exchange integral $j_{\text {eff }}=J_{\text {eff }} / J$ vs. $t$ for $S=1 / 2,1$, and $5 / 2$; different values of the anisotropy parameter $\mu=0.9,0.5$, and 0 are also shown by the dashed, full and dotted curves, respectively.

In Fig. 2, the ratio $\left(\mu_{\mathrm{eff}}-\mu\right) / \mu$ is plotted against $\mu$ for different spin and temperature values: the quantum renormalization is here seen to more strongly affect the most anisotropic models, due to the fact that, when forced to align along the easy axis, each spin reacts with larger quantum fluctuations perpendicular to the axis itself.

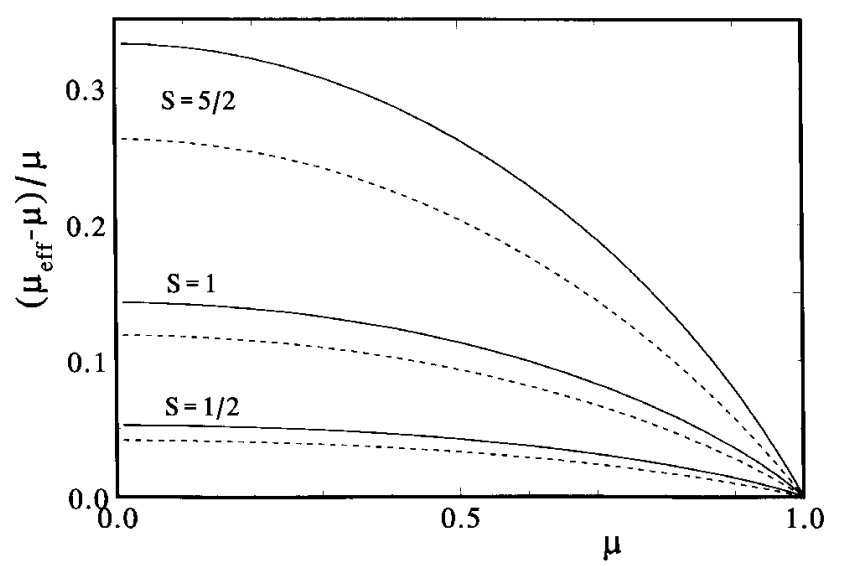

Figure 2. Ratio $\left(\mu_{\mathrm{eff}}-\mu\right) / \mu$ vs. $\mu$ for $S=1 / 2,1$, and $5 / 2$; different temperature values $t=0.001$ and 0.1 are shown by the full and dashed curves, respectively.

From Eq. (7) we see that the symmetry properties of the original quantum Hamiltonian are preserved by the renormalization procedure leading to the effective Hamiltonian: this means that the critical behaviour of the EA-QHAF can be directly related to that of its effective classical counterpart. In particular, if $t_{\mathrm{c}}^{\mathrm{cl}}(\mu)$ is the reduced critical temperature of a classical EA-HAF with anisotropy parameter $\mu$, then, for the quantum system, it is

$$
t_{\mathrm{c}}(S, \mu)=\frac{t_{\mathrm{c}}^{\mathrm{cl}}\left(\mu_{\mathrm{eff}}\left(t_{\mathrm{c}}\right)\right)}{j_{\mathrm{eff}}\left(t_{\mathrm{c}}\right)} .
$$

For fixed $S$ and $\mu$, the $t$ dependence of $j_{\text {eff }}$ and $\mu_{\text {eff }}$ make Eq. (8) rather complicated. Its solution, however, can be obtained iteratively, once $t_{c}^{\mathrm{cl}}(\mu)$ is available as an analytical function of $\mu$. To obtain such a function we used the classical Monte Carlo data reported in Refs. [13] and [14]: these data are well distributed over the interval $0 \leq \mu<1$ and an accurate interpolating function $\tau$ may hence be determined. In particular we have used

$$
\tau_{\mathrm{c}}^{\mathrm{cl}}(\mu)=a /\left[1+b \mu^{2}+c \mu^{4}-d \ln \left(1-\mu^{2}\right)\right],
$$

with $a=0.917, b=0.068, c=0.097$, and $d=0.0636$. The logarithmic dependence upon $\left(1-\mu^{2}\right)$ is required in order to properly describe the logarithmic drop of $t_{\mathrm{c}}^{\mathrm{cl}}$ for $\mu \rightarrow 1$; $\tau_{\mathrm{c}}^{\mathrm{cl}}$ has been set an even function of $\mu$ 
to ensure the ferro- and antiferromagnetic models to be equivalent, as required for the static behaviour of classical magnetic systems.

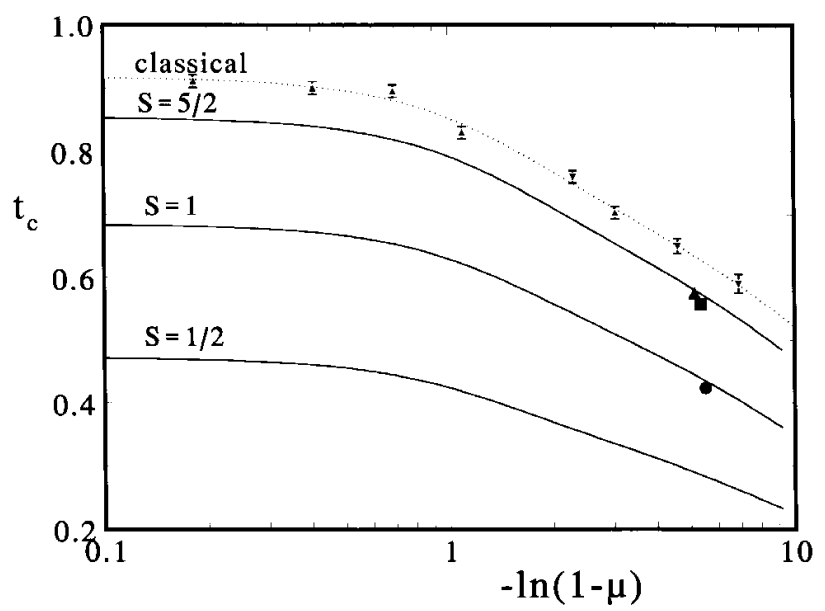

Figure 3. Critical temperature $t_{\mathrm{c}}(S, \mu)$ of the EA-QHAF vs. $-\ln (1-\mu)$ for $S=1 / 2,1,5 / 2$, and $\infty$ (classical model). Large symbols indicate the experimentally determined critical temperatures for $\mathrm{Rb}_{2} \mathrm{MnF}_{4}$ (triangle), $\mathrm{K}_{2} \mathrm{MnF}_{4}$ (square), and $\mathrm{K}_{2} \mathrm{NiF}_{4}$ (circle). Small symbols are classical Monte Carlo data from Refs. [13] and [14], upwards and downwards triangles, respectively. The dotted curve is the interpolating function $\tau_{\mathrm{c}}^{\mathrm{cl}}(\mu)$ (see text)

In Fig. 3 the resulting solution of Eq. (8) is plotted against $-\ln (1-\mu)$ to expand the $\mu \simeq 1$ region, where data for real compounds are available; the agreement between our curves and the experimentally determined critical temperatures is very good for both $S=1$ and $S=5 / 2$, given also the fact that no best-fit procedure is involved in the comparison. On the other hand, the exactly known [15] critical temperature of the Ising model $t_{c}(1 / 2,0)=0.567$, not represented in Figure, is not well reproduced in view of the large values of the renormalization coefficients Eq. (5), and we expect our results to be only qualitatively significant in the extreme $S=1 / 2$ quantum case. This respect, we cannot give a definite answer to the problem, recently addressed in Ref. [4], of the existence of a finite $\mu$-interval, near the isotropic limit $\mu=1$, where $t_{c}(1 / 2, \mu)=0$, i.e. where the EA-
QHAF shows no finite-temperature transition.

In conclusion, we have studied the EA-QHAF in terms of the effective Hamiltonian determined by the PQSCHA method; by analyzing the critical temperature dependence on the anisotropy parameter $\mu$, the Ising-like phase-transition characterizing the model has been found to occur at finite temperature $\forall \mu<1$ and $\forall S \geq 1$; a qualitative indication in favour of the validity of this result for $S=1 / 2$ has also been obtained.

\section{References}

[1] M. Bander and D. L. Mills, Phys. Rev. B 38, 12015 (1988).

[2] L. P. Kadanoff et al., Rev. Mod. Phys. 39, 395 (1967).

[3] J. A. Hertz, Phys. Rev. B 14, 1165 (1976).

[4] N. S. Branco and J. Ricardo de Sousa, preprint, condmat/0006345 (June 2000).

[5] J. Kanamori, in Magnetism vol.1, ed. G.T.Rado e H.Suhl, Academic Press, New York (1963).

[6] R. A. Cowley, G. Shirane, R. J. Birgeneau, H. J. Guggenheim, Phys. Rev. B 15, 4292 (1977).

[7] R. J. Birgeneau, H. J. Guggenheim, G. Shirane, Phys. Rev. B 8, 304 (1973).

[8] H. W. de Wijn, L. R. Walker, R. E. Walstedt, Phys. Rev. B 8, 285 (1973).

[9] A. Cuccoli, V. Tognetti, P. Verrucchi and R. Vaia, Phys. Rev. A 45, 8418 (1992).

[10] A. Cuccoli, R. Giachetti, V. Tognetti, R. Vaia, and P. Verrucchi, J. Phys., Condens. Matter 7, 7891 (1995).

[11] A. Cuccoli, V. Tognetti, R. Vaia, and P. Verrucchi Phys. Rev. B 46, 11601 (1992).

[12] A. Cuccoli, V. Tognetti, R. Vaia, and P. Verrucchi Phys. Rev. Lett. 77, 3439 (1996), Phys. Rev. B 56, 14456 (1997).

[13] P. A. Serena, N. García and A. Levanyuk, Phys. Rev. B 47, 5027 (1993).

[14] M. E. Gouvêa el al., Phys. Rev. B 59, 6229 (1999).

[15] L. Onsager, Phys. Rev. 65, 117 (1944). 